\title{
A Simple Method to Derive the Bistatic Tracking Radar System Formulas
}

\author{
Khalil Ibrahim Ali Al-Sharabi \\ College of Electronics Engineering \\ University of Mosul
}

\begin{abstract}
Bistatic Tracking Radar (BTR) is an advanced mode of conventional Bistatic Radar (BR) which is used for target detection. The principle of working of BTR system is same as that for Mono Static Tracking Radar (MTR), with the difference that the transmitter is apart from the receiver, and they operate separately and independently. In this paper, the associated equations that control the BTR system operation, was derived in a simple manner. These equations determine the receiver and transmitter antenna positions to keep their lines of sight meet at the target, beside the assurance of tracking the target during maneuvering. The operation of the system was explained and the simulation program was made to characterize the operation of the system by using the derived equations in this paper.
\end{abstract}

Keywords: Bistatic Radar, Tracking, Radar

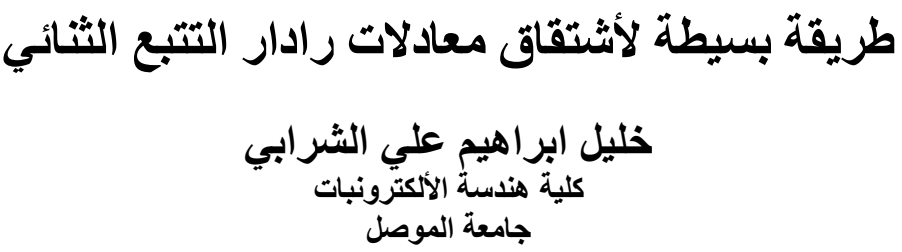

الخلاصة الزائة

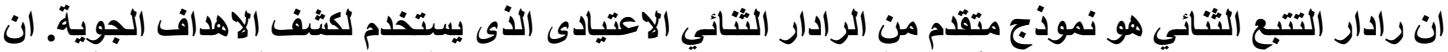

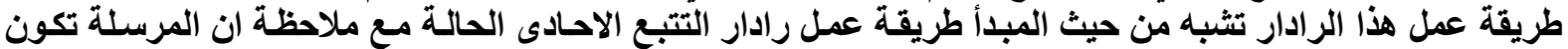

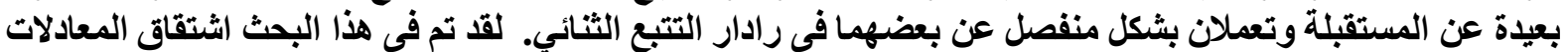

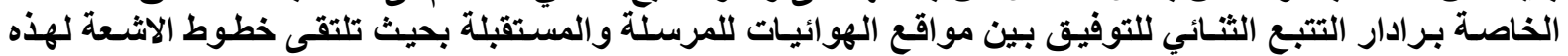

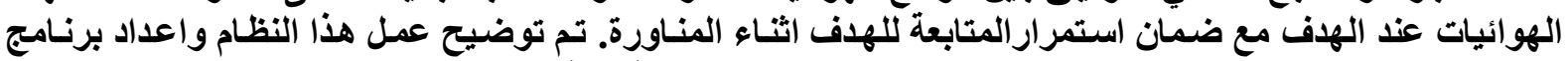

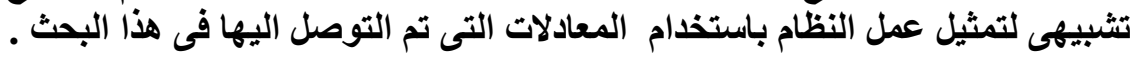




\section{Introduction}

In general the bistatic Radar is a radar system in which the transmitter and the receiver are separated by a distance comparable to the target-to-receiver range used for target detection, positioning, and tracking [1,2].The Monostatic radars suffer from countermeasures, such as jamming, and anti-radiation weapons, while the bistatic radars have less vulnerability to countermeasures[3,4].This is because the countermeasures are concentrated toward the transmitter which is far from the receiver that is keeping the receiver out of threat. The implementation of the bistatic radar is more complex than the monostatic radar because it needs synchronization for time, frequency, and antenna positioning [5]. Many researches have been made to solve these problems such as in references $[6,7,8,9]$. In addition, the equation of bistatic radar is more complex than that of monostatic radar. This paper interests with Bistatic Tracking Radar (BTR), which is the most complex mode of the bistataic radar systems. This radar is mostly used in military applications, especially for air defense under the warfare environments, so that, it has the highest technology for accurate operation. The bistatic radar equation given by Skolnik [1], Willis [2], and Schejbal [3], determines the general bistatic range equation applied for bistatic radar systems. This equation is not sufficient for bistatic tracking radar because it needs antenna (or spatial) synchronization. In this paper, the study was concentrated to derive the equations that represent the relationships between the transmitter antenna and the receiver antenna directions to control the movement of the transmitter antenna relative to the movement of the tracking receiver antenna to make the system work compatibly. The principle of operation of the system is the master-slave with high communication channel. The receiver station is the master station, which is equipped by the tracking receiver, the data processing, and control units, while transmitter station is the slave which receives the commands from the receiver station to steer its antenna toward the target as shown in figure (1).

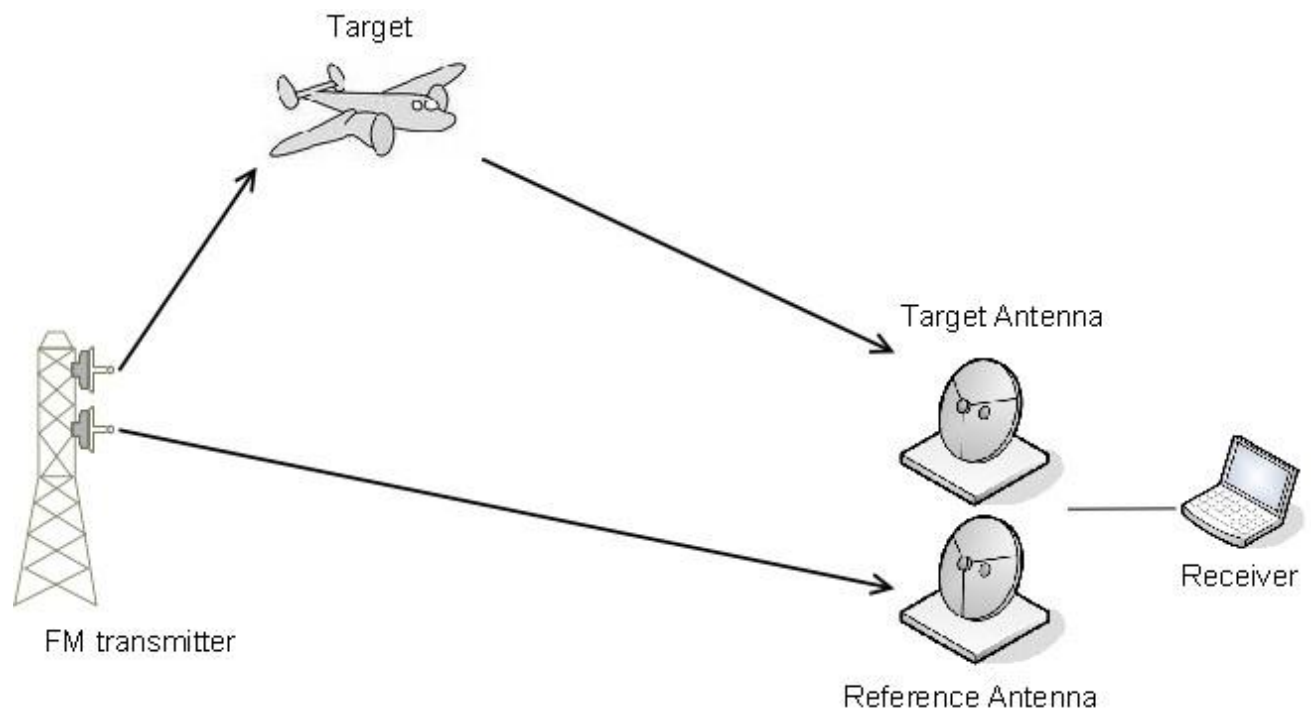

Fig. (1) Bistatic radar stations and their locations

The initial data of the target representing the elevation angle, the azimuth angle, and the range of the target, are given with respect to the receiver station. In the receiver, the information will be processed by using the formulas of operation of the BTR system that will be derived later, and the commands will send to the transmitter to steer its antenna toward the target. Through the tracking phase, the commands are continuously send to the transmitter to 
keep its antenna illuminating the target. The triangulation method was used to derive the BTR formulas as explained later.

\section{Geometrical Characteristics}

In the bistatic radar where the target, the transmitter, and the receiver are separated from each other, and each one is far from others. Therefore, the simplest method to analyze the geometry of the system is the triangulation method which conventional to derive angular relationships between parts of the system. In this method, the target, the transmitter, and the receiver make heads of a triangle as shown in figure (2). In this figure, the heads of the triangle $(\mathrm{T}, \mathrm{R}$, and $\mathrm{O})$ represent the transmitter, the receiver, and the target respectively.

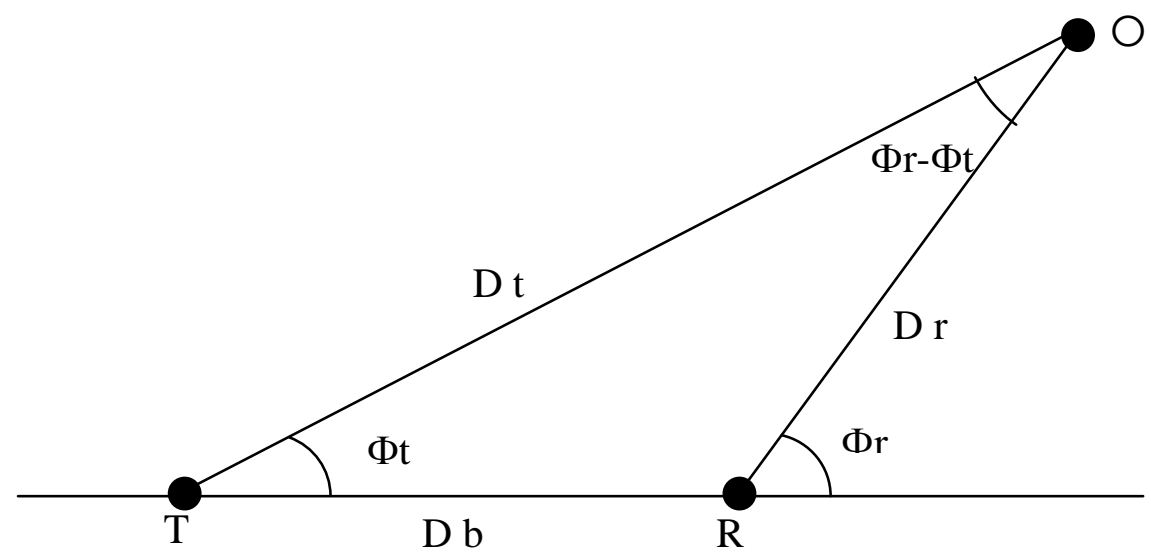

Fig. (2) Bistatic triangle geometry

From figure (2), the three geometrical parameters which are considered as the source variables are:

a. The transmitter-receiver antenna separation: this is known as the Base Distance $\left(D_{b}\right)$, which represents the distance between transmitter and receiver. This distance must be constant during the operation of the system.

b. The Transmitter-Target-receiver distance (S): this distance is known as the Triangulation Distance which is represented by the summation of the transmitter-target distance $\left(D_{t}\right)$, and the target-receiver distance $\left(D_{r}\right)$. It depends on the target position, and it varies due to the continuous moving of the target.

c. The Transmitter-Target-Receiver angle $(\Phi r-\Phi t)$ : this is the main parameter of the (BTR) geometry that gives the satisfactory of system operation, and it is known as the Bistatic Angle. This angle is represented by the intersection of the transmitter-target sight line, and the receiver-target sight line. This angle equals the difference between the transmitter and receiver antenna direction angles $\left(\Phi_{\mathrm{r}}-\Phi_{\mathrm{t}}\right)$, and it determines the triangulation factor of the bistatic radar $(\mathrm{F})$ where:

$$
\mathrm{F}=\sin \left(\left(\Phi_{\mathrm{r}}-\Phi_{\mathrm{t}}\right) / 2\right)
$$

For real bistatic characteristics and operation, this factor must be in the range of (0.1-0.9) as shown in Fig.(3) [5]. The formulas to obtain values of $\left(\Phi_{r}\right.$, and $\left.\Phi_{t}\right)$ are given in appendix $A$ 


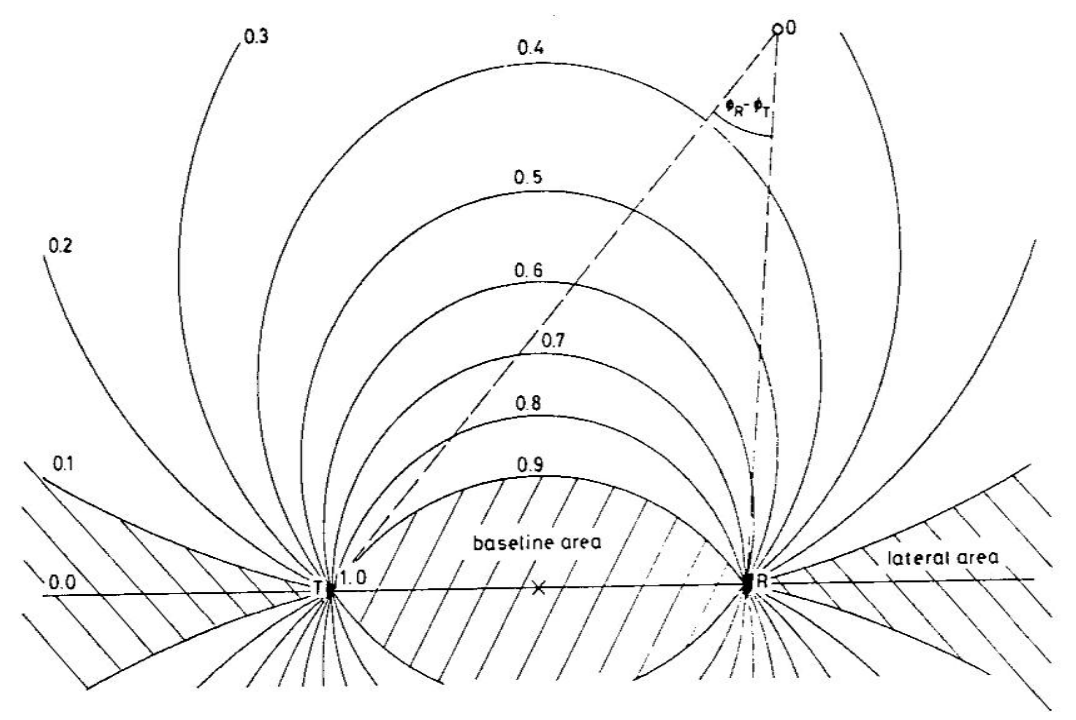

Fig. (3) Triangulation factor for bistatic radar

\section{Bistatic Formulas and Geometry}

The transmitted signal reaches the receiver after being reflected from the target through the bistatic (triangular) path between transmitter-target-receiver. Since the distance between transmitter and receiver is large and each one of them operates independently, several parameters must be taken into account. The measured parameters are considered as primary such as the base distance between transmitter and receiver, the delay time which is known as propagation time of the signal representing the bistatic path (distance), the receiver antenna direction (including azimuth, and elevation angles), with respect to the receiver station. The other parameters are calculated by using the (BTR) formulas to perform operation of the system. The formulas of (BTR) are defined in the plane of the triangle with vertices represented by transmitter $(\mathrm{T})$, receiver $(\mathrm{R})$, and target $(\mathrm{O})$. To simplify the derivation of (BTR) formulas this approach considers the projection of this triangle on the ground defined by the heads as transmitter $(\mathrm{T})$, receiver $(\mathrm{R})$, and the projection of target on the ground (P) as shown in Fig.(4). Figure (4) shows that, the projection of the target is out of the line connecting the transmitter and the receiver as a general condition because the target is mostly either to the left or right of this line.

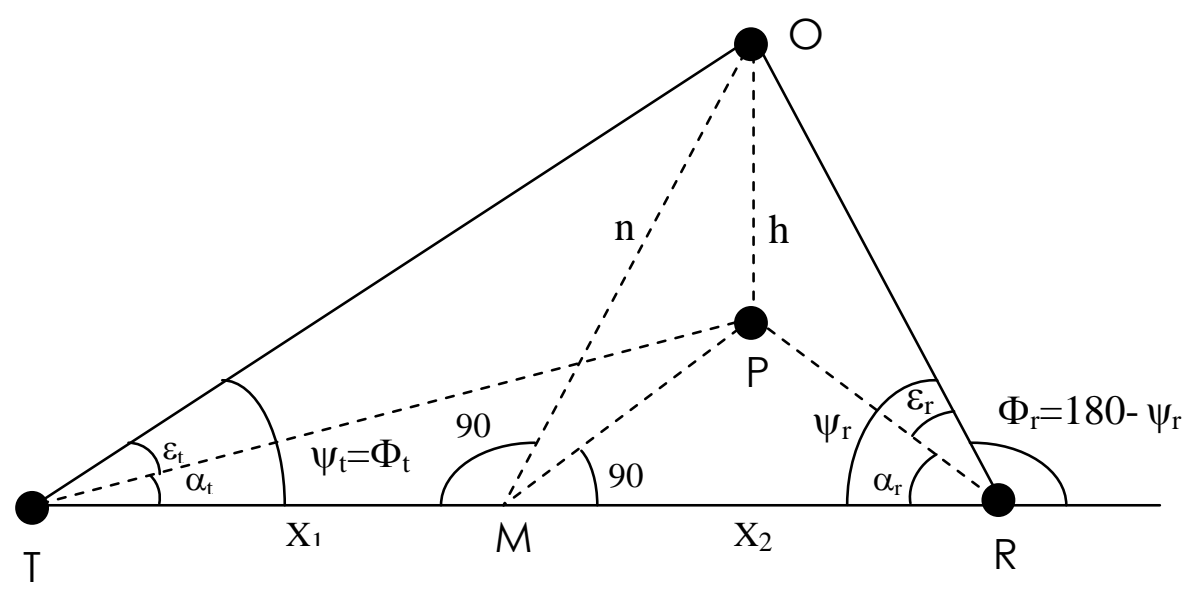

Fig.(4) Detailed Bistatic radar geometry 
The parameters of the (BTR) and their associated formulas are defined as in the following:

a. The transmitter-receiver distance $\left(D_{b}\right)$ : this is the base distance which can be measured by using the navigation method such as the Global Positioning System (GPS) to determine the locations of transmitter and receiver stations.

b. The transmitter-target-receiver distance: this is called the triangulation distance, the path length, or the bistatic path which can be determined by measuring the time of arrival (delay time) of the transmitted signal after being reflected from the target to reach the receiver, and it is given as:

$\mathrm{S}=\mathrm{T} \times \mathrm{C}$

Where $(\mathrm{C})$ is the speed of light, $(\mathrm{T})$ is the delay time between transmitted and received signal, and (S) is the bistatic distance or the triangular distance which represents the transmitter-target-receiver path of the ray. The accurate method for measuring the delay time is by using (GPS) timing signal to synchronize the timing operation between transmitter and receiver stations.

c. The receiver antenna direction $\left(\psi_{\mathrm{r}}\right)$ : this is the angle which represents the direction of the reflected signal from the target to the receiver. This angle can be accurately calculated in reading the elevation and azimuth angles from the antenna positioning mechanism, and using the formula:

$\psi_{\mathrm{r}}=\cos ^{-1}\left(\cos \varepsilon_{\mathrm{r}} \cos \alpha_{\mathrm{r}}\right)$

In this formula $\left(\psi_{\mathrm{r}}\right)$ represents the direction of receiver antenna toward the target, $\left(\varepsilon_{\mathrm{r}}\right)$ is the elevation angle of the receiver antenna, and $\left(\alpha_{\mathrm{r}}\right)$ is the azimuth angle of the receiver antenna. Note that $\left(\varepsilon_{\mathrm{r}}\right.$ and $\left.\alpha_{\mathrm{r}}\right)$ angles can be taken directly from the positioning mechanism of the receiver antenna. The derivation of the receiver antenna direction in equation (3) is given in appendix A.

d. The target-receiver distance $\left(D_{r}\right)$ : it represents the distance between the target and the receiver. This distance can be calculated by using the following formula [10], which is derived in appendix $\mathrm{B}$ :

$$
D_{r}=\frac{S^{2}-D_{b}{ }^{2}}{2\left(S-D_{b} \cos \left(\Psi_{r}\right)\right.}
$$

e. The target-transmitter distance $\left(D_{t}\right)$ : it represents the distance between the target and the transmitter. This distance can be calculated using the following formula which is derived in appendix B:

$$
D_{t}=\frac{S^{2}+D_{b}{ }^{2}-2 S D_{b} \cos \left(\Psi_{r}\right)}{2\left(S-D_{b} \cos \left(\Psi_{r}\right)\right)}
$$

f. Transmitter antenna direction $\left(\psi_{\mathrm{t}}\right)$ : it is the combination of azimuth and elevation angles of the transmitter antenna. These angles can be calculated according to the receiver antenna azimuth and elevation angle. Therefore the elevation angle of transmitter antenna can be calculated using the following formula:

$$
\varepsilon_{t}=\sin ^{-1} \frac{D_{r} \sin \left(\varepsilon_{r}\right)}{S-D_{r}}
$$


And the azimuth angle of the transmitter antenna can be calculated using the following formula:

$$
\alpha_{t}=\sin ^{-1} \frac{D_{r} \cos \left(\varepsilon_{r}\right) \sin \left(\alpha_{r}\right)}{\left(S-D_{r}\right) \cos \left(\varepsilon_{t}\right)}
$$

Derivation of equations (6) and (7) is given in appendix C.

The direction of transmitter antenna $\left(\psi_{t}\right)$ can be calculated in the same manner as used to derive the receiver antenna direction formula, which is given in appendix A. This direction is given by the following formula:

$\psi_{\mathrm{t}}=\cos ^{-1}\left(\cos \left(\varepsilon_{\mathrm{t}}\right) \cos \left(\alpha_{\mathrm{t}}\right)\right)$

\section{System Operation}

The scenario of the system operation can be summarized by receiving the initial data of the target, locking the target, and tracking of the target. The locations of the transmitter and receiver stations can be determined by using GPS, or the contour maps for the region of operation, and fixed in the post station (Detection Radar Station). The post station provides data of the target with respect to the receiver and transmitter locations including $\left(\varepsilon_{\mathrm{r}}, \alpha_{\mathrm{r}}, \varepsilon_{\mathrm{t}}\right.$, $\alpha_{t}, D_{r}, D_{t}$, and $D_{b}$ ) and send them to the receiver station, as initial data. These data are processed and examined in the computer of the system which exists in the receiver station. When the transmitter and receiver antennas are directed toward the target as shown in figure (5), the system starts operation and the transmitter illuminates the target and the computer in the receiver will compare the measured data with the incoming data from the post station. The measured data are obtained by reading the transmitter and receiver antenna positions, and the time delay between the direct signal from the transmitter to the receiver for coherent receiver, or by using the GPS timing signal as reference time for transmit and receive operation for the noncoherent receiver. The time delay $(\mathrm{T})$ provides the triangulation distance (S) between Transmitter-Target-Receiver. As the lock signal exists the receiver will continue to track the target and send commands (including $\varepsilon_{t}$ and $\alpha_{t}$ ) to the transmitter, antenna to illuminate the target .During the track phase, the computer reads the position of the receiver antenna ( $\varepsilon_{\mathrm{r}}$ and $\alpha_{\mathrm{r}}$ ), and the time delay, then calculates the triangulation distance (S), and the transmitter antenna position ( $\varepsilon_{\mathrm{t}}$ and $\alpha_{\mathrm{t}}$ ) by using the derived equations. This commands transferred to the transmitter station by using telecommunication system accurately.

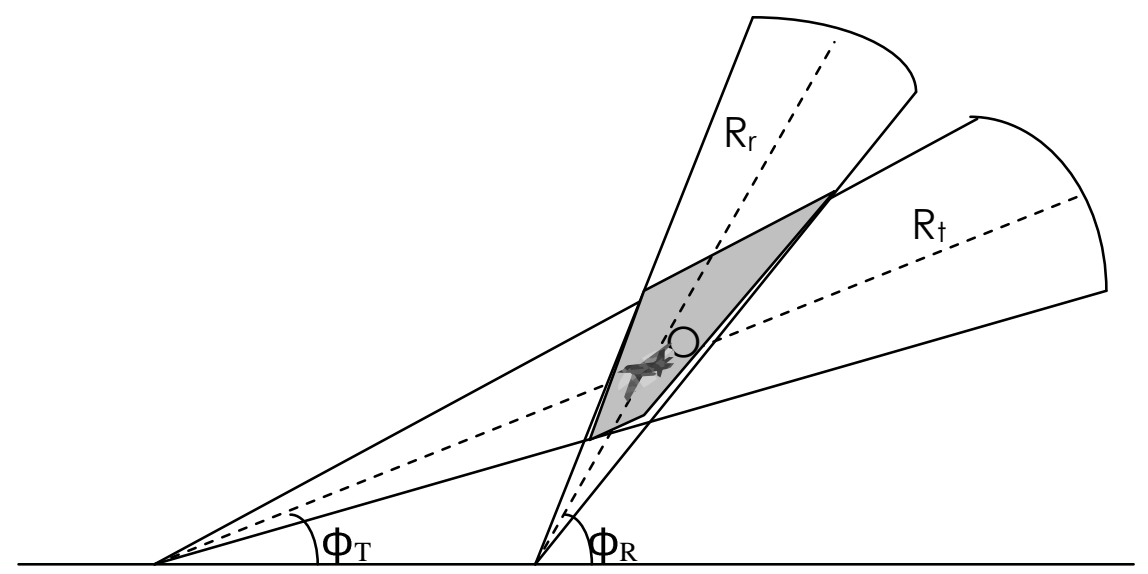

Fig.(5) Intersection of transmitter and receiver antenna main lobs at the target. 


\section{Simulation Results}

To validate the proposed method of deriving the BTR system equations and algorithm, we present the simulation results to provide the utility of this method. In the simulation, three different cases of the initial information about the target were taken. The first check will be done by checking the value of the triangulation factor ( F ) in equation ( 1 ) to identify the condition of the BTR system whether it is valid or not. If it is not valid, the system will reset, for valid condition the system will proceed to check the Lock On signal state. If the lock signal is (1) the system will proceed and tracking is performed for the maneuvering target. If the lock signal is (0), the system will

reset. The simulation of the bistatic tracking radar geometry was developed by using (MATLAB 7) program. The program was designed to provide the validity of operation of the system before starting operations. Also the program can be used with interface to calculate the geometry during the tracking phase as much as the target is locked by the tracking receiver. During the tracking phase, the program calculates the position of the transmitter antenna relative to the position of the receiver antenna to make the center lines of the transmitter and receiver antenna main lobs intersected at the target and to keep the intersection continuous during maneuvering of the target. The simulation results for such assumed conditions of the target are listed in table (1). The flow chart shown in figure (6) illustrates the steps to compute the position of the transmitter antenna relative to the position of the

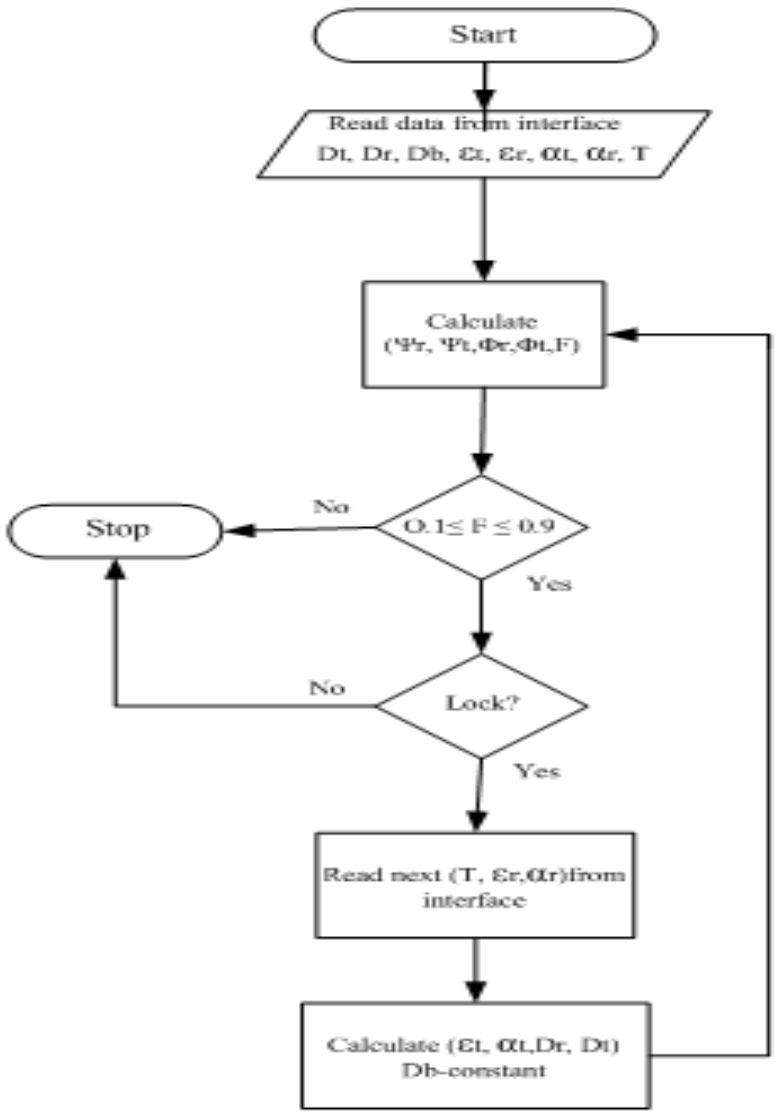
receiver antenna,

Fig. (6) Flow chart of simulation program

Table (1) Simulation results for the BTR system

\begin{tabular}{|c|c|c|c|c|c|c|c|c|c|c|c|c|c|}
\hline \multicolumn{7}{|c|}{ Input Data } & \multicolumn{7}{|c|}{ Output Data } \\
\hline $\begin{array}{c}\varepsilon \mathrm{r} \\
\mathrm{deg}\end{array}$ & $\begin{array}{c}\alpha \mathrm{r} \\
\mathrm{deg}\end{array}$ & $\begin{array}{c}\varepsilon \mathrm{t} \\
\mathrm{deg}\end{array}$ & $\begin{array}{c}\alpha \mathrm{t} \\
\mathrm{deg}\end{array}$ & $\begin{array}{r}\mathrm{Dr} \\
\mathrm{Km}\end{array}$ & $\begin{array}{c}\mathrm{Dt} \\
\mathrm{Km}\end{array}$ & $\begin{array}{c}\mathrm{Db} \\
\mathrm{Km}\end{array}$ & $\begin{array}{r}\Phi r \\
\text { deg }\end{array}$ & $\begin{array}{r}\Phi t \\
\text { deg }\end{array}$ & $\mathrm{F}$ & $\begin{array}{c}\mathrm{T} \\
\mu \mathrm{sec}\end{array}$ & $\begin{array}{c}\mathrm{S} \\
\mathrm{Km}\end{array}$ & $\mathrm{L}$ & status \\
\hline 27 & 26 & 13 & 11 & 5 & 10.5 & 14 & 143.25 & 17 & 0.88 & 52 & 15.6 & 1 & Valid \\
\hline 27 & 26 & 10 & 8 & 5 & 14.5 & 10 & 36.7 & 12.3 & 0.21 & 64.3 & 19.3 & 1 & valid \\
\hline 170 & 350 & 6 & 4 & 10 & 30 & 20 & 14 & 6 & 0.07 & $\mathrm{X}$ & $\mathrm{X}$ & $\mathrm{X}$ & invalid \\
\hline
\end{tabular}

\section{Accuracy and error sources}

From table (1), it can be seen that there is no error in the numerical calculations of the system geometry, because the system takes the real values of data and use them in the derived equations to calculate the relative transmitter antenna direction (ie. $\varepsilon_{t}$, and $\alpha_{t}$ ). The accuracy of the system depends on the accuracy of equipments used in the implementation, and the 
error is created by the measuring equipments, but not from the equations applied in the numerical calculations. The resultant error appears in the direction of the transmitter antenna. To overcome the effect of the resultant error from the system devices, the Half Power Beam Width (HPBW) of the transmitter antenna must be larger than that of the receiver antenna to keep illumination on the moving target during tracking phase. For such system, the accepted value of error is about $(1 \%-5 \%)$ depending on the accuracy of the system equipments and costs.

\section{Conclusion}

In this paper, the concept of bistatic tracking radar was explained by using a transmitter and tracking receiver. This paper was concentrated in the triangulation method for the derivation of the bistatic tracking radar formulas in order to solve the problem of antenna direction synchronization between the transmitter and the receiver. The derived equations in this paper were examined sequentially by using MATLAB program, so this sequence could be applied in the software implementation to control the process of the system operation. The proposed triangulation factor for such BTR system could be taken in the range (0.2-0.8) as a special condition for the military applications.

Applying this method can solve the problem of geometry complexity of the bistatic tracking radar as well as simplify the control operations, and synchronization between transmitter and receiver antenna. In other words this approach can solve many difficulties in the practical implementation and operation of this type of radar system by using the relationships between the transmitter and receiver antenna positions.

\section{Acknowledgements}

The author would like to thank Dr.Museeb M. Jasem, and Dr. Thaffer Al Neema, for their valuable comments, and suggestions.

\section{References}

1. Merril I. Skolnik : "Radar Handbook", 1970, pp (36.1-36.18).

2. N. J. Willis, and H., D., Griffiths : "Advances in Bistatic Radar", 2007, pp(23.123.36).

3. P.Bezousek, V. Schejbal : "Bistitic and Multistatic Radar Systems" ,Radioengineering, Vol.17, No.3,Sept. 2008, pp(53-59).

4. NAWCWPNS-TP 8347: "Electronic Warefare and Radar System Engineering Hand Book", 1999, pp(4-4.1-4-8.5).

5. E. Hanle : "Survey of bistatic and multistatic radar", IEE proceedings, Vol.132, Pt.F, No. 7,Dec. 1986, pp. (587-595).

6. D.Madurasinghe and A.P. Shaw :"Target Localization by Resolving the Time Synchronization Problem in Bistatic Radar Systems using Space Fast-Time Adaptive Processor", EURASIP Journal on Advance in Signal Processing, Vol. 2009, pp(1-17).

7. W.Q. Wang and J. Cai:" Antenna Directing Synchronization for Bistatic Synthetic Aperture Radar Systems.", IEEE Antenna and Wireless Propagation Letters, Vol. 9, 2010,pp(307-310).

8. W. Q. Wang: " GPS-Based Time, and Phase Synchronization Processing for Distributed SAR",IEEE Transaction on Aerospace and Electronic Systems",Vol. 45, No. 3, July. 2009, pp (1040-1051).

9. Aadil Volkwin: "Suitability of a Commercial Software Defined Radio System for Passive Coherent Location.", A dissertation Submitted to the Department of Electrical 
Engineering, University of Cape Tawn, In fulfillment of requirement for degree of Master of Science in Engineering. May- 2008, (chp. 2).

10. Merrill.I.Skolnik "Introduction to Radar Systems",1980, pp. (553-560).

\section{Appendices}

\section{Appendix A}

Derivation of receiver antenna and transmitter antenna direction formulas:

From the triangle (OPR) in figure (4):

$$
\mathrm{OP}=\mathrm{D}_{\mathrm{r}} \sin \varepsilon_{\mathrm{r}}
$$

$\mathrm{PR}=\mathrm{D}_{\mathrm{r}} \cos \varepsilon_{\mathrm{r}}$

From triangle MPR

$$
\mathrm{MR}=\mathrm{PR} \cos \alpha_{\mathrm{r}}
$$

Substituting the value of (PR) from equation (2) then equation (3) will be $\mathrm{MR}=\mathrm{D}_{\mathrm{r}} \cos \varepsilon_{\mathrm{r}} \cos \alpha_{\mathrm{r}}$

From triangle OMR

$$
\mathrm{MR}=\mathrm{D}_{\mathrm{r}} \cos \psi_{\mathrm{r}}
$$

Combining equations (4) and (5) then

$$
\begin{aligned}
& \cos \psi_{\mathrm{r}}=\cos \varepsilon_{\mathrm{r}} \cos \alpha_{\mathrm{r}} \\
& \psi_{\mathrm{r}}=\cos ^{-1}\left(\cos \varepsilon_{\mathrm{r}} \cos \alpha_{\mathrm{r}}\right) \\
& \Phi_{\mathrm{r}}=180-\psi_{\mathrm{r}}
\end{aligned}
$$

In the same manner the direction of transmitter antenna formula will be given as

$$
\begin{aligned}
& \psi_{\mathrm{t}}=\cos ^{-1}\left(\cos \varepsilon_{\mathrm{t}} \cos \alpha_{\mathrm{t}}\right) \\
& \Phi_{\mathrm{t}}=\psi_{\mathrm{t}}
\end{aligned}
$$

\section{Appendix B}

Derivation of receiver-target and transmitter-target distance formulas:

From the triangle (OMR) in figure (4)

$$
\begin{gathered}
\text { Let } \mathrm{OM}=\mathrm{n}, \mathrm{MR}=\mathrm{X}_{2} \\
\mathrm{D}_{\mathrm{r}}{ }^{2}=\mathrm{n}^{2}+\mathrm{X}_{2}{ }^{2} \\
\mathrm{~N}^{2}=\mathrm{D}_{\mathrm{r}}{ }^{2}-\mathrm{X}_{2}{ }^{2}
\end{gathered}
$$

From triangle OMT

$$
\begin{aligned}
& \text { Let } \mathrm{MT}=\mathrm{X}_{1} \\
& \mathrm{D}_{\mathrm{t}}{ }^{2}=\mathrm{n}^{2}+\mathrm{X}_{1}{ }^{2} \\
& \mathrm{~N}^{2}=\mathrm{D}_{\mathrm{t}}{ }^{2}-\mathrm{X}_{1}{ }^{2}
\end{aligned}
$$

Combining equations (2) and (4) then

$$
\mathrm{D}_{\mathrm{t}}^{2}-\mathrm{X}_{1}^{2}=\mathrm{D}_{\mathrm{r}}{ }^{2}-\mathrm{X}_{2}^{2}
$$

But $\quad \mathrm{X}_{1}=\mathrm{D}_{\mathrm{b}}-\mathrm{X}_{2}$ then

$$
\begin{aligned}
& \mathrm{D}_{\mathrm{t}}{ }^{2}-\left(\mathrm{D}_{\mathrm{b}}-\mathrm{X}_{2}\right)^{2}=\mathrm{D}_{\mathrm{r}}{ }^{2}-\mathrm{X}_{2}{ }^{2} \\
& \mathrm{D}_{\mathrm{t}}{ }^{2}-\mathrm{D}_{\mathrm{b}}{ }^{2}+2 \mathrm{D}_{\mathrm{b}} \mathrm{X}_{2}-\mathrm{X}_{2}{ }^{2}=\mathrm{D}_{\mathrm{r}}{ }^{2}-\mathrm{X}_{2}{ }^{2} \\
& \mathrm{D}_{\mathrm{t}}{ }^{2}-\mathrm{D}_{\mathrm{b}}{ }^{2}+2 \mathrm{D}_{\mathrm{b}} \mathrm{X}_{2}=\mathrm{D}_{\mathrm{r}}{ }^{2}
\end{aligned}
$$

By adding the term $\left(\mathrm{D}_{\mathrm{r}}^{2}+2 \mathrm{D}_{\mathrm{r}} \mathrm{D}_{\mathrm{t}}\right)$ to both side of equation (8) then

$$
\mathrm{D}_{\mathrm{t}}^{2}+2 \mathrm{D}_{\mathrm{r}} \mathrm{D}_{\mathrm{t}}+\mathrm{D}_{\mathrm{r}}{ }^{2}-\mathrm{D}_{\mathrm{b}}{ }^{2}+2 \mathrm{D}_{\mathrm{b}} \mathrm{X}_{2}=\mathrm{D}_{\mathrm{r}}{ }^{2}+\mathrm{D}_{\mathrm{r}}{ }^{2}+2 \mathrm{D}_{\mathrm{r}} \mathrm{D}_{\mathrm{t}}
$$

$\left(\mathrm{D}_{\mathrm{t}}+\mathrm{D}_{\mathrm{r}}\right)^{2}-\mathrm{D}_{\mathrm{b}}^{2}+2 \mathrm{D}_{\mathrm{b}} \mathrm{X}_{2}=2 \mathrm{D}_{\mathrm{r}}^{2}+2 \mathrm{D}_{\mathrm{r}} \mathrm{D}_{\mathrm{t}}$

$\mathrm{S}^{2}-\mathrm{D}_{\mathrm{r}}^{2}+2 \mathrm{D}_{\mathrm{b}} \mathrm{X}_{2}=2 \mathrm{D}_{\mathrm{r}}^{2}+2 \mathrm{D}_{\mathrm{r}} \mathrm{D}_{\mathrm{t}}$
$\mathrm{S}^{2}-\mathrm{D}_{\mathrm{b}} \mathrm{D}_{\mathrm{r}}^{2}+2 \mathrm{D}_{\mathrm{t}} \mathrm{D}_{\mathrm{t}}-2 \mathrm{D}_{\mathrm{b}} \mathrm{X}_{2}$

$S^{2}-D_{b}^{2}=2 D_{r}^{2}+2 D_{r} D_{t}-2 D_{b} X_{2}$

But $\mathrm{X}_{2}=\mathrm{D}_{\mathrm{r}} \cos \psi_{\mathrm{r}}$

Then $S^{2}-D_{b}^{2}=2 D_{r}^{2}+2 D_{r} D_{t}-2 D_{b} D_{r} \cos \psi_{r}$

$\mathrm{S}^{2}-\mathrm{D}_{\mathrm{b}}^{2}=2 \mathrm{D}_{\mathrm{r}}\left(\mathrm{D}_{\mathrm{r}}+\mathrm{D}_{\mathrm{t}}-\mathrm{D}_{\mathrm{b}} \cos \psi_{\mathrm{r}}\right)$

$\mathrm{S}^{2}-\mathrm{D}_{\mathrm{b}}^{2}=2 \mathrm{D}_{\mathrm{r}}\left(\mathrm{S}-\mathrm{D}_{\mathrm{b}} \cos \psi_{\mathrm{r}}\right)$

Therefore $\mathrm{D}_{\mathrm{r}}=\frac{S^{2}-D_{b}{ }^{2}}{2\left(S-D_{b} \cos \psi_{r}\right)}$

The formula for the distance between the transmitter and the target derived as following. 


$$
\begin{aligned}
& \mathrm{D}_{\mathrm{t}}=\mathrm{S}-\mathrm{D}_{\mathrm{r}} \\
& \mathrm{D}_{\mathrm{t}}=S-\frac{S^{2}-D_{b}{ }^{2}}{2\left(S-D_{b} \cos \psi_{r}\right)} \\
& \text { Then } \mathrm{D}_{\mathrm{t}}=\frac{S^{2}+D_{b}{ }^{2}-2 S D_{b} \cos \psi_{r}}{2\left(S-D_{b} \cos \psi_{r}\right)}
\end{aligned}
$$

\section{Appendix C}

Derivation of transmitter antenna elevation and azimuth angles formulas:

From the triangle (OPR) in figure (4)

$\mathrm{OP}=\mathrm{D}_{\mathrm{r}} \sin \varepsilon_{\mathrm{r}}$

$\mathrm{PR}=\mathrm{D}_{\mathrm{r}} \cos \varepsilon_{\mathrm{r}}$

From triangle OPT

$\mathrm{OP}=\mathrm{D}_{\mathrm{t}} \sin \varepsilon_{\mathrm{t}}$

$\mathrm{PT}=\mathrm{D}_{\mathrm{t}} \cos \varepsilon_{\mathrm{t}}$

And $\mathrm{D}_{\mathrm{t}}=\mathrm{S}-\mathrm{D}_{\mathrm{r}}$

Then equations (3) and (4) will be written as

$$
\begin{aligned}
& \mathrm{OP}=\left(\mathrm{S}-\mathrm{D}_{\mathrm{r}}\right) \sin \varepsilon_{\mathrm{t}} \\
& \mathrm{PT}=\left(\mathrm{S}-\mathrm{D}_{\mathrm{r}}\right) \cos \varepsilon_{\mathrm{t}}
\end{aligned}
$$

Combining equations (1) and (6)

$\left(\mathrm{S}-\mathrm{D}_{\mathrm{r}}\right) \sin \varepsilon_{\mathrm{t}}=\mathrm{D}_{\mathrm{r}} \sin \varepsilon_{\mathrm{r}}$

Then $\varepsilon_{\mathrm{t}}=\sin ^{-1} \frac{D_{r} \sin \varepsilon_{r}}{S-D_{r}}$

From triangle PMR

$$
\mathrm{MP}=\mathrm{PR} \sin \alpha_{\mathrm{r}}
$$

Substituting the value of PR from equation (2)

$$
\mathrm{MP}=\mathrm{D}_{\mathrm{r}} \cos \varepsilon_{\mathrm{r}} \sin \alpha_{\mathrm{r}}
$$

From triangle MPT

$$
\mathrm{MP}=\mathrm{PT} \sin \alpha_{\mathrm{t}}
$$

Substituting the value of PT from equation (7) then

$$
\mathrm{MP}=\left(\mathrm{S}-\mathrm{D}_{\mathrm{r}}\right) \cos \varepsilon_{\mathrm{t}} \sin \alpha_{\mathrm{t}}
$$

Substituting the value of MP from equation (12) then

$\left(\mathrm{S}-\mathrm{D}_{\mathrm{r}}\right) \cos \varepsilon_{\mathrm{t}} \sin \alpha_{\mathrm{t}}=\mathrm{D}_{\mathrm{r}} \cos \varepsilon_{\mathrm{r}} \sin \alpha_{\mathrm{r}}$

$\sin \alpha_{\mathrm{t}}=\frac{D_{r} \cos \varepsilon_{r} \sin \alpha_{r}}{\left(S-D_{r}\right) \cos \varepsilon_{t}}$

$\alpha_{\mathrm{t}}=\sin ^{-1} \frac{D_{r} \cos \varepsilon_{r} \sin \alpha_{r}}{\left(S-D_{r}\right) \cos \varepsilon_{t}}$

\section{The work was carried out at the College of Electronics Engineering} University of Mosul 